\title{
$f$-CLEAN RINGS AND RINGS HAVING MANY FULL ELEMENTS
}

\author{
Binguun Li And Lianggui Feng
}

\begin{abstract}
An associative ring $R$ with identity is called a clean ring if every element of $R$ is the sum of a unit and an idempotent. In this paper, we introduce the concept of $f$-clean rings. We study various properties of $f$-clean rings. Let $C=\left(\begin{array}{cc}A & V \\ W & B\end{array}\right)$ be a Morita Context ring. We determine conditions under which the ring $C$ is $f$-clean. Moreover, we introduce the concept of rings having many full elements. We investigate characterizations of this kind of rings and show that rings having many full elements are closed under matrix rings and Morita Context rings.
\end{abstract}

\section{Introduction}

Clean rings were introduced by Nicholson [14]. An element $r$ in a ring $R$ is said to be clean if it can be written as the sum of a unit and an idempotent in $R$. A ring $R$ is called a clean ring if every element of $R$ is clean. Such rings constitute a subclass of exchange rings in the theory of noncommutative rings. Following Nicholson [14], $R$ is an exchange ring if and only if for any element $a$ in $R$ there exists an idempotent $e$ in $R$ such that $e \in R a$ and $1-e \in R(1-a)$. Semiperfect rings and unit-regular rings are examples of clean rings as shown by Camillo and $\mathrm{Yu}$ [5] and Camillo and Khurana [4]. In recent years, many authors have studied clean rings and their generalizations such as $[1,13,16,17]$.

In this paper, we extend clean rings and introduce the concept of $f$-clean rings. Examples of $f$-clean rings are given. We study various properties of $f$-clean rings. We prove that $M_{n}(R)$ is $f$-clean for any $f$-clean ring $R$ and get a condition under which the definitions of cleanness and $f$-cleanness are equivalent.

A Morita Context $(A, B, V, W, \psi, \phi)$ consists two rings $A, B$, two bimodules ${ }_{A} V_{B},{ }_{B} W_{A}$ and a pair of bimodule homomorphisms $\psi: V \otimes_{B} W \rightarrow A, \phi:$

Received May 6, 2008; Revised January 12, 2009.

2000 Mathematics Subject Classification. Primary 16D70; 16 U99.

Key words and phrases. full elements, $f$-clean rings, matrix rings, rings having any full elements.

This research is supported by NCET(06-0923) and NUDT(JC0803) and the Research Foundation of Education Department of Hunan Province. 
$W \otimes_{A} V \rightarrow B$, such that $\psi(v \otimes \omega) v^{\prime}=v \phi\left(\omega \otimes v^{\prime}\right), \phi(\omega \otimes v) \omega^{\prime}=\omega \psi\left(v \otimes \omega^{\prime}\right)$. We can form

$$
C=\left\{\left(\begin{array}{cc}
a & v \\
\omega & b
\end{array}\right) \mid a \in A, b \in B, v \in V, \omega \in W\right\}
$$

and define a multiplication on $C$ as follows:

$$
\left(\begin{array}{ll}
a & v \\
\omega & b
\end{array}\right)\left(\begin{array}{cc}
a^{\prime} & v^{\prime} \\
\omega^{\prime} & b^{\prime}
\end{array}\right)=\left(\begin{array}{cc}
a a^{\prime}+\psi\left(v \otimes \omega^{\prime}\right) & a v^{\prime}+v b^{\prime} \\
\omega a^{\prime}+b \omega^{\prime} & \phi\left(\omega \otimes v^{\prime}\right)+b b^{\prime}
\end{array}\right) .
$$

A routine check shows that, with this multiplication (and entry-wise addition), $C$ becomes an associative ring. We call $C$ a Morita Context ring. Obviously, the class of the rings of Morita Contexts includes all $2 \times 2$ matrix rings and all formal triangular matrix rings. In recent years, many authors studied Morita Contexts from different points of view [6, 12]. We obtain the relationship of $f$-cleanness between Morita Context ring $C$ and $A, B$.

Definition 1.1. An element $x \in R$ is said to be a full element if there exist $s, t \in R$ such that $s x t=1$. The set of all full elements of a ring $R$ will be denoted by $K(R)$. Obviously, invertible elements and one-sided invertible elements are all in $K(R)$.

Definition 1.2. An element in $R$ is said to be $f$-clean if it can be written as the sum of an idempotent and a full element. A ring $R$ is called a $f$-clean ring if each element in $R$ is a $f$-clean element.

Example 1.3. The notion of purely infinite simple rings was introduced by Ara, Goodearl, and Pardo [3]. A simple unital ring $R$ is purely infinite in case that it is not a division ring and for each nonzero element $x$ in $R$, there exist elements $z, t$ in $R$ such that $z x t=1$. The class of purely infinite simple rings is quite large, one can find various examples in [3, Example 1.3]. Ara [2, Theorem 1.1] proved that every purely infinite simple ring is an exchange ring. We do not know whether every purely infinite simple ring is an clean ring. But for any $x$ in a purely infinite simple ring, we have $x=0$ or $x \in K(R)$. Hence, every purely infinite simple ring is a $f$-clean ring.

A ring $R$ is said to have stable range one provided that for any $a, b \in R$, $a R+b R=R$ implies that there exists some $y \in R$ such that $a+b y \in U(R)$, where $U(R)$ denotes the set of all units in $R$. We say that $R$ satisfies unit 1stable range provided that $a R+b R=R$ implies that there exists some $u \in U(R)$ such that $a+b u \in U(R)$. Many authors have studied this class of rings such as $[9,11]$. In Section 3, we generalize the concept of rings having unit 1-stable range to rings having many full elements: A ring $R$ is said to have many full elements provided that $a R+b R=R$ implies that there exists some $\omega \in K(R)$ such that $a+b \omega \in U(R)$. We investigate various characterizations of this kind of rings. We show that $A, B$ have many full elements, so have $M_{n}(A)$ for any $n \geq 1$ and the Morita Context ring $C$ as defined above. 
Throughout this paper all rings are assumed to be associative with identity and modules are unitary. $J(R)$ always stands for the Jacobson radical of a ring $R$. $M_{n}(R)$ denotes the $n \times n$ matrix ring over the ring $R . T_{n}(R)$ stands for $n \times n$ upper triangular matrix ring. The notation $R^{n \times 1}$ always stands for the set

$$
\left\{\left(\begin{array}{c}
x_{1} \\
\vdots \\
x_{n}
\end{array}\right) \mid x_{1}, \ldots, x_{n} \in R\right\},
$$

which is an $\left(M_{n}(R), R\right)$-bimodule. The notation $R^{1 \times n}$ stands for the set $\left\{\left(x_{1}, \ldots, x_{n}\right) \mid x_{1}, \ldots, x_{n} \in R\right\}$, which is an $\left(R, M_{n}(R)\right)$-bimodule. The transpose of a matrix $A$ will be denoted by $A^{T}$. $G L_{n}(R)$ denotes the general linear group of $R^{n}$. Let $B_{i j}(x)=I_{2}+x e_{i j}(i \neq j, 1 \leq i, j \leq 2),[\alpha, \beta]=\alpha e_{11}+\beta e_{22}$, where $e_{i j}$ are matrix units and $\alpha, \beta \in U(R) . \pi: R \rightarrow R / J(R)$ will denote the natural quotient ring homomorphism from $R$ to $R / J(R)$, and we will write $\pi(r)=\bar{r}$ and $\bar{R}=R / J(R)$.

\section{2. $f$-clean rings}

Firstly, we get some basic properties of $f$-clean rings.

Proposition 2.1. (1) Any homomorphic image of a $f$-clean ring is $f$-clean.

(2) A direct product $R=\prod R_{i}$ of rings $\left\{R_{i}\right\}$ is $f$-clean if and only if the same is true for each $R_{i}$.

Proof. (1) is straightforward.

(2) Suppose that each $R_{i}$ is a $f$-clean ring. For any $x=\left(x_{i}\right) \in R$ and each $i$, we write $x_{i}=e_{i}+w_{i}$ with $e_{i}^{2}=e_{i}$ and $s_{i} w_{i} t_{i}=1$ for some $s_{i}, t_{i} \in R$. Then $x=e+w$, where $e=\left(e_{i}\right)$ is an idempotent in $\prod R_{i}$ and $w=\left(w_{i}\right) \in \prod R_{i}$ with $\left(s_{i}\right)\left(w_{i}\right)\left(t_{i}\right)=(1) \in \prod R_{i}$. Hence $x$ is $f$-clean, as required. The converse follows from (1).

It was proved by Camillo and $\mathrm{Yu}[5]$ that the $\operatorname{ring} R$ is a clean ring if and only if $\bar{R}$ is clean and idempotents can be lifted modulo $J(R)$. We do not know whether idempotents in $f$-clean ring can be lifted modulo $J(R)$. But we have the following:

Proposition 2.2. Let $R$ be a ring. If idempotents can be lifted modulo $J(R)$, then $R$ is a f-clean ring if and only if $\bar{R}$ is a f-clean ring.

Proof. One direction is trivial and does not depend on the hypotheses by Proposition 2.1(1).

Conversely, suppose that $\bar{R}$ is a $f$-clean ring. Let $x \in R$, then $\bar{x}=\bar{e}+\bar{w}$ with $e^{2}-e \in J(R)$ and $\bar{s} \bar{w} \bar{t}=\overline{1}$ for some $s, t \in R$. Since idempotents can be lifted modulo $J(R)$, we may assume $e$ is an idempotent and write $x=e+w+r$ for some $r \in J(R)$. Again, we have $s w t=1+h \in 1+J(R) \subseteq U(R)$ for some $h \in J(R)$. Therefore, there exist $s_{1}, t_{1} \in R$ such that $s_{1} w t_{1}=1$. Hence 
$s_{1}(w+r) t_{1}=1+s_{1} r t_{1} \in 1+J(R) \subseteq U(R)$. We have $s_{1}(w+r) t_{1} u^{-1}=1$ for some $u \in U(R)$, hence $w+r$ is a full element. Therefore, $x$ is $f$-clean, as asserted.

Anderson and Camillo [1] showed that every polynomial ring over a nonzero commutative ring is not clean. Full elements and invertible elements are the same when the ring $R$ is commutative, so the concepts of clean ring and $f$-clean ring are equivalent for commutative rings. Hence every polynomial ring over a nonzero commutative ring is also not $f$-clean. But the following holds:

Proposition 2.3. Let $R$ be a f-clean ring. If idempotents can be lifted modulo $J(R)$, then $R[x] /\left\langle x^{n+1}\right\rangle$ is a $f$-clean ring for any $n \geq 1$.

Proof. Assume that $R$ is a $f$-clean ring. Denote $\bar{x}=x+\left\langle x^{n+1}\right\rangle$ in $R[x] /\left\langle x^{n+1}\right\rangle$ by $u$, then $R[x] /\left\langle x^{n+1}\right\rangle=R[u]=R+R u+\cdots+R u^{n}$ with $u^{n+1}=0$. It is not difficult to prove that $J(R[u])=J(R)+\langle u\rangle$, where $\langle u\rangle$ denotes the ideal of $R[u]$ generated by $u$. Hence $R[u] / J(R[u]) \simeq \bar{R}$ is $f$-clean by Proposition 2.1 .

Now we claim that idempotents can be lifted modulo $J(R[u])$. Let $f+$ $J(R[u])$ be an idempotent in $R[u] / J(R[u])$, then $f=a_{0}+a_{1} u+\cdots+a_{n} u^{n}$ for some $a_{0}, a_{1}, \ldots, a_{n} \in R$. Note that $J(R[u])=J(R)+\langle u\rangle$, thus $\left(a_{0}+\right.$ $J(R[u]))^{2}=a_{0}^{2}+J(R[u])=a_{0}+J(R[u])$. Hence $a_{0}^{2}-a_{0} \in J(R)$. Since idempotents can be lifted modulo $J(R)$, there exists $e=e^{2} \in R \subseteq R[u]$ such that $e-a_{0} \in J(R) \subseteq J(R[u])$. Thus $f$ can be lifted to $e$. The result follows from Proposition 2.1.

Conversely, assume that $R[u]$ is $f$-clean. $R[u] / J(R[u]) \simeq \bar{R}$ shows that $R / J(R)$ is $f$-clean. Note that idempotents can be lifted modulo $J(R)$ by assumption, hence we get that $R$ is $f$-clean by Proposition 2.2 .

Recall that for a ring $R$ with a ring endomorphism $\alpha: R \rightarrow R$, the skew power series ring $R[[x ; \alpha]]$ of $R$ is the ring obtained by giving the formal power series ring over $R$ with the new multiplication $x r=\alpha(r) x$ for all $r \in R$. In particular, $R[[x]]=R\left[\left[x, 1_{R}\right]\right]$.

Proposition 2.4. Let $\alpha$ be an endomorphism of $R$. Then the following statements are equivalent.

(1) $R$ is a f-clean ring.

(2) The formal power series ring $R[[x]]$ of $R$ is a $f$-clean ring.

(3) The skew power series ring $R[[x ; \alpha]]$ of $R$ is a $f$-clean ring.

Proof. Being an image of $R[[x]]$ and $R[[x ; \alpha]], R$ is $f$-clean when $R[[x]]$ or $R[[x ; \alpha]]$ is $f$-clean.

$(1) \Rightarrow(3)$ For any $h=a_{0}+a_{1} x+\cdots \in R[[x, \alpha]]$, write $a_{0}=e_{0}+u_{0}$ for some $e_{0}=e_{0}^{2}, u_{0} \in K(R)$. Assume that $s_{0} u_{0} t_{0}=1$ for some $s_{0}, t_{0} \in R$ and let $h^{\prime}=h-e_{0}=u_{0}+a_{1} x+\cdots$. The equation $u=\left(s_{0}+0+\cdots\right) h^{\prime}\left(t_{0}+0+\right.$ $\ldots)=1+s_{0} a_{1} \alpha\left(t_{0}\right) x+\cdots$ shows that $u \in U(R[[x, \alpha]])$, since $U(R[[x ; \alpha]])=$ $\left\{a_{0}+a_{1} x+\cdots: a_{0} \in U(R)\right\}$ without any assumption on the endomorphism $\alpha$. Hence $h^{\prime} \in K(R[[x, \alpha]])$ and $h=e_{0}+h^{\prime}$ with $e_{0}^{2}=e_{0} \in R[[x, \alpha]]$. 
$(1) \Rightarrow(2)$ Since $R[[x]]=R\left[\left[x, 1_{R}\right]\right]$, the proof is similar to that of $(1) \Rightarrow(3)$, as desired.

Camillo and Khurana [4] showed that any unit-regular ring is clean. Han and Nicholson [13] proved that every (finite) matrix over a clean ring is clean.

Theorem 2.5. If $R$ is a $f$-clean ring, then $M_{n}(R)$ is also a $f$-clean ring for any $n \geq 1$.

Proof. Suppose that $R$ is $f$-clean. Given any $x \in R$, we have some idempotent $e$ and $w \in K(R)$ such that $x=e+w$. We write $s w t=1$ for some $s, t \in R$.

Assume that theorem holds for the matrix ring $M_{k}(R), k \geq 1$.

Let

$$
A=\left(\begin{array}{ll}
a_{11} & a_{12} \\
a_{21} & a_{22}
\end{array}\right) \in M_{k+1}(R)
$$

with $a_{11} \in R, a_{12} \in R^{1 \times k}, a_{21} \in R^{k \times 1}$ and $a_{22} \in M_{k}(R)$.

We have $a_{11}=e+w$ with $e=e^{2}$ and $s w t=1$ for some $s, t \in R$. There also exist an idempotent matrix $E$ and a full matrix $W$ such that $a_{22}-a_{21} t s a_{12}=$ $E+W$ by hypothesis. We write $S W T=I_{k}$ for some $S, T \in M_{k \times k}(R)$.

Therefore, we have

$$
A=\operatorname{diag}(e, E)+\left(\begin{array}{cc}
w & a_{12} \\
a_{21} & W+a_{21} t s a_{12}
\end{array}\right) .
$$

Obviously, $\operatorname{diag}(e, E)$ is an idempotent matrix in $M_{k+1}(R)$.

Let

$$
P=\left(\begin{array}{cc}
s & 0 \\
-S a_{21} t s & S
\end{array}\right), Q=\left(\begin{array}{cc}
t & -t s a_{12} T \\
0 & T
\end{array}\right) \in M_{k+1}(R),
$$

and the equation

$$
P\left(\begin{array}{cc}
w & a_{12} \\
a_{21} & W+a_{21} t s a_{12}
\end{array}\right) Q=\left(\begin{array}{cc}
1 & 0 \\
0 & I_{n}
\end{array}\right)=I_{k+1}
$$

shows that $\left(\begin{array}{cc}w & a_{12} \\ a_{21} & W+a_{21} t s a_{12}\end{array}\right)$ is a full matrix, hence $A$ is $f$-clean, as desired.

Proposition 2.6. If $a \in R$ is a $f$-clean element, then $A=\left(\begin{array}{ll}a & b \\ 0 & 0\end{array}\right)$ is always $f$-clean in $M_{2}(R)$ for any $b \in R$.

Proof. If $a=e+w$ where $e=e^{2}$ and $s w t=1$ for some $s, t \in R$. Then we can write $A$ as

$$
A=\left(\begin{array}{ll}
e & 0 \\
0 & 1
\end{array}\right)+\left(\begin{array}{cc}
w & b \\
0 & -1
\end{array}\right) .
$$

We also have $\left(\begin{array}{cc}s & 0 \\ 0 & -1\end{array}\right)\left(\begin{array}{cc}w & b \\ 0 & -1\end{array}\right)\left(\begin{array}{cc}t & -t s b \\ 0 & 1\end{array}\right)=\left(\begin{array}{ll}1 & 0 \\ 0 & 1\end{array}\right)$, hence $\left(\begin{array}{cc}w & b \\ 0 & -1\end{array}\right)$ is a full element. Therefore, $A$ is a $f$-clean element.

Let $C$ be the Morita Context ring as defined in Section 1. We are going to investigate the $f$-cleanness between Morita Context ring $C$ and $A, B$. Our concern here is the Morita Context rings with zero homomorphisms. 
Theorem 2.7. Let $C=\left(\begin{array}{cc}A & V \\ W & B\end{array}\right)$ be the Morita Context with $\psi, \varphi=0$. Then $C$ is $f$-clean if and only if $A$ and $B$ are $f$-clean.

Proof. Assume that $C$ is $f$-clean with $\psi, \varphi=0$, let $I=\left(\begin{array}{cc}0 & V \\ W & B\end{array}\right), J=\left(\begin{array}{cc}A & V \\ W & 0\end{array}\right)$. One can check that $I, J$ are ideals of $C$ and $C / I \simeq A, C / J \simeq B$. The $f$-cleanness of $A, B$ follows from Proposition 2.1.

Conversely, let $A, B$ both $f$-clean rings. For any $r=\left(\begin{array}{ll}a & 0 \\ w & b\end{array}\right) \in C$, we have $a=e_{1}+u_{1}$ and $b=e_{2}+u_{2}$ for some idempotents $e_{1}, e_{2} \in R$ and $u_{1}, u_{2} \in$ $K(R)$. Assume that $s_{1} u_{1} t_{1}=1, s_{2} u_{2} t_{2}=1$ for some $s_{1}, t_{1}, s_{2}, t_{2} \in R$. Let $r=\left(\begin{array}{cc}e_{1} & 0 \\ 0 & e_{2}\end{array}\right)+\left(\begin{array}{cc}u_{1} & v \\ \omega & u_{2}\end{array}\right)=E+U$. Obviously, $E=E^{2}$ and the equation

$$
\left(\begin{array}{cc}
s_{1} & 0 \\
-s_{2} \omega t_{1} s_{1} & s_{2}
\end{array}\right)\left(\begin{array}{cc}
u_{1} & v \\
\omega & u_{2}
\end{array}\right)\left(\begin{array}{cc}
t_{1} & -t_{1} s_{1} v t_{2} \\
0 & t_{2}
\end{array}\right)=\left(\begin{array}{ll}
1 & 0 \\
0 & 1
\end{array}\right)
$$

implies that $U$ is a full matrix. Hence $r$ is $f$-clean, as required.

Proposition 2.8. (1) Let $R, S$ be two rings, and $M$ be an $(R, S)$-bimodule. Let $E=\left(\begin{array}{cc}R & M \\ 0 & S\end{array}\right)$ be the formal triangular matrix ring. Then $E$ is $f$-clean ring if and only if $R$ and $S$ are $f$-clean rings.

(2) For any $n \geq 1, R$ is $f$-clean if and only if the $n \times n$ upper triangular matrix ring $T_{n}(R)$ are $f$-clean.

Proof. Formal triangular matrix rings are special cases of the Morita Context rings with zero morphisms, thus (1) follows by Theorem 2.7.

Let $E, A \in T_{n}(R)$. It is straightforward to calculate that $E^{2}=E$ then $E_{i i}^{2}=E_{i i}$, and $A \in K\left(T_{n}(R)\right)$ if and only if $A_{i i} \in K(R)$, where $E_{i i}, A_{i i}$ are matrix units, $1 \leq i \leq n$. Hence (2) is also straightforward.

Next we will investigate the equivalence of $f$-cleanness and cleanness. Yu [18] call a ring $R$ to be a left quasi-duo ring if every maximal left ideal of $R$ is a two-sided ideal. Commutative rings, local rings, rings in which every nonunit has a power that is central are all belong to this class of rings [18]. A ring $R$ is said to be Dedekind finite if $x y=1$ always implies $y x=1$ for any $x, y \in R$. A ring $R$ is called abelian if all idempotents are central.

Theorem 2.9. For a left quasi-duo ring $R$, the following are equivalent:

(1) $R$ is a clean ring;

(2) $R$ is a $f$-clean ring.

Proof. (1) $\Rightarrow(2)$ is trivial.

(2) $\Rightarrow$ (1) It suffices to show that $\omega \in K(R)$ implies that $\omega \in U(R)$. Let $s \omega t=1$ for some $s, t \in R$, so $s$ is right invertible. Assume that $s$ is not left invertible. Then $R s \neq R$, and there exists a maximal left ideal $M$ of $R$ such that $R s \subseteq M \neq R$. Therefore, $M$ is an ideal by assumption. Note that $s \in M$, thus we get that $s R \subseteq M$, contradicting the fact that $s$ is right invertible. Hence we get $\omega t s=1$, which shows that $\omega$ is a right invertible element. In a similar way, we get that $\omega \in U(R)$, and the result follows.

Corollary 2.10. Every abelian $f$-clean ring is a clean ring. 
Proof. It suffices to prove that every abelian ring is Dedekind finite by the proof Theorem 2.9. Suppose $a b=1$, then $b a$ is an idempotent and hence central by assumption. $b a=b a \cdot a b=a b \cdot a b=1$ shows that $R$ is Dedekind finite. Then the result follows from the proof of Theorem 2.9 .

Let $R$ be a ring in which 2 is invertible, Camillo and Yu [5] showed that $R$ is clean if and only if every element of $R$ is the sum of a unit and a square root of 1 . We get an analogous result for $f$-clean rings. If $G$ is a group, we denote the group ring over $R$ by $R G$. It seems to be difficult to characterize $R$ and $G$ for which $R G$ is $f$-clean in general. We here also focus on $R$ with $2 \in U(R)$.

Proposition 2.11. Let $R$ be a ring in which 2 is invertible and $G=\{1, g\}$ be a group.

(1) $R$ is $f$-clean if and only if every element of $R$ is the sum of a full element and a square root of 1 .

(2) $R G$ is $f$-clean if and only if $R$ is $f$-clean.

Proof. (1) Suppose $R$ is $f$-clean and $x \in R$. Then $(x+1) / 2=e+u$ for some $e^{2}=e$ and $u \in K(R)$. So $x=(2 e-1)+2 u$ with $(2 e-1)^{2}=1$ and $2 u \in K(R)$.

Conversely, if every element of $R$ is the sum of a full element and a square root of 1 . Then given $x \in R$, we have $2 x-1=t+w$ with $t^{2}=1$ and $w$ a full element in $R$. So $x=(t+1) / 2+w / 2$ with $((t+1) / 2)^{2}=(t+1) / 2$ and $w / 2$ a full element in $R$, as asserted.

(2) As an image of $R G, R$ is $f$-clean when $R G$ is $f$-clean. Conversely, Since $2 \in U(R)$, we have $R G \simeq R \times R$ via the map $\theta: a+b g \mapsto(a+b, a-b)[13$, Proposition 3]. Hence $R G$ is $f$-clean by Proposition 2.1 .

For an idempotent $e$, we do not know whether the corner ring $e R e$ is again $f$-clean for a $f$-clean ring $R$. But when $e$ is a central idempotent, we can get an affirmative answer:

Proposition 2.12. Let $R$ be a $f$-clean ring and e be a central idempotent in $R$. Then eRe is also $f$-clean.

Proof. We can view $e R e$ as a homomorphic image of $R$ since $e$ is central, hence the result follows from Proposition 2.1.

Let $R$ be a ring and ${ }_{R} V_{R}$ be an $R$-R-bimodule which is a ring possibly without a unity in which $(v \omega) r=v(\omega r),(v r) \omega=v(r \omega)$ and $(r v) \omega=r(v \omega)$ hold for all $v, \omega \in V$ and $r \in R$. The ideal extension of $R$ by $V$ is defined to be the additive abelian group $I(R, V)=R \oplus V$ with multiplication $(r, v)(s, \omega)=$ $(r s, r \omega+v s+v \omega)$.

Proposition 2.13. An ideal-extension $E=I(R, V)$ of $R$ by $V$ is $f$-clean if $R$ is $f$-clean and for any $v \in R$, there exists $\omega \in R$ such that $v+\omega+\omega v=0$.

Proof. Let $s=(r, v) \in E$. Then $r=e+u$ for some $e=e^{2}, u \in K(R)$. $s=(e, 0)+(u, v)$. Obviously, $(e, 0)$ is an idempotent in $E$, and we will show that 
$(u, v) \in K(E)$. Assume that $s u t=1$. For $s v t \in V$, there exists $\omega \in V$ such that $s v t+\omega+\omega s v t=0$ by assumption, and one can check that $(s, \omega s)(u, v)(t, 0)=1$. Hence $(u, v) \in K(E)$ and $E$ is a $f$-clean ring.

\section{Rings having many full elements}

Recall that a ring $R$ is said to have stable range one provided that $a R+b R=$ $R$ implies that there exists some $y \in R$ such that $a+b y \in U(R)$. The most vital property of rings having stable range one is that stable range one in endomorphism rings implies cancellation in direct sums: $A, B, C$ are $R$-modules such that $A \oplus B=A \oplus C$, and $\operatorname{End}_{R}(A)$ has stable range one, then $B \simeq C$.

Goodearl and Mental [11] defined the concept of unit 1-stable range: Whenever $a, b \in R$ satisfy $a R+b R=R$, there exists a unit $u \in R$ such that $a+b u$ is a unit. By replacing $u \in U(R)$ with unit-regular element, Chen [10] defined the concept of rings with many unit-regular elements, and investigated various properties of this class of rings.

Given an idempotent $e$ in $R$, we say that $x \in R$ is $e$ - $f$-clean if $x$ can be written in the form $e+\omega$ for some $\omega \in K(R)$. Although this idempotent is not uniquely determined by $x$, it can help us to classify $f$-clean elements. Obviously, elements in $K(R)$ are exactly 0 - $f$-clean elements. In this section, we introduce a new class of rings: rings having many full elements.

Firstly, we give some elementary properties of full elements, which will be used repeatedly later.

Lemma 3.1. The following hold for any ring $R$.

(1) If $\omega \in K(R)$, then $u \omega$ and $\omega u$ are both in $K(R)$ for any $u \in U(R)$.

(2) $\omega \in K(R)$ if and only if $\bar{\omega} \in K(\bar{R})$.

Proof. (1) Given $\omega \in K(R)$ and $u \in U(R)$, there exist $s, t \in R$ such that $s \omega t=1$. Then $s u^{-1} u \omega t=1$ and $s \omega u u^{-1} t=1$, which imply that $u \omega$ and $\omega u$ are both in $K(R)$.

(2) Given $\omega \in K(R)$, there exist $s, t \in R$ such that $s \omega t=1$. Thus $\overline{s \omega t}=\overline{1} \in$ $\bar{R}$. Conversely, Given $\bar{\omega} \in K(\bar{R})$, there exist $\bar{s}, \bar{t} \in \bar{R}$ such that $\overline{s \omega t}=\overline{1}$. Hence $s \omega t=1+r \in U(R)$ for some $r \in J(R)$. Thus $s \omega t(1+r)^{-1}=1$, showing that $\omega \in K(R)$

Proposition 3.2. The following statements are equivalent for any ring $R$ :

(1) Whenever $a, b \in R$ satisfy $a R+b R=R$, there exists $\omega \in K(R)$ such that $a+b \omega \in U(R)$.

(2) Whenever $a, b \in R$ satisfy $a R+b R=R$, there exists $\omega \in K(R)$ such that $a+b \omega$ is left invertible.

(3) Whenever $a, b \in R$ satisfy $a R+a R=R$, there exists $\omega \in K(R)$ such that $a+\omega b$ is right invertible.

If $R$ satisfies any of these conditions, we say that $R$ has many full elements.

Proof. (1) $\Rightarrow(2)$ and (1) $\Rightarrow(3)$ are trivial. 
$(3) \Rightarrow(1)$ Given $a R+b R=R$, then $a+b \omega=u$ is right invertible for some $\omega \in K(R)$ by assumption. Say $u v=1$ for some $v \in R$.

Since $v R+(1-v u) R=R$, there is $\omega_{1} \in K(R)$ such that $v+(1-v u) \omega_{1}=\omega_{2}$ is right invertible.

It is easy to see that $u \omega_{2}=u\left(v+(1-v u) \omega_{1}\right)=1$, which implies that $\omega_{2}$ is a unit in $R$. Thus $u=a+b \omega$ is a unit.

$(2) \Rightarrow(1)$ Given $a R+b R=R$, then $a+b \omega=u$ is left invertible for some $\omega \in K(R)$ by assumption. Say $v u=1$ for some $v \in R$.

$v R+0 R=R$ implies that we can find $\omega_{1} \in K(R)$ such that $v+0 \omega_{1}=v$ is left invertible. Hence $a+b \omega \in U(R)$, as asserted.

In view of the proof of Theorem 2.9, if $R$ is a quasi-duo ring, then $R$ has many full elements if and only if $R$ satisfies unit 1-stable range.

The following two propositions are analogous to [17, Lemma 4.4, Theorem 4.5]. The proofs are also similar, so we omit them here.

Proposition 3.3. Let $R$ be a ring. Then the following are equivalent:

(1) $R$ has many full elements.

(2) Whenever $a, b \in R$ satisfy $a x+b=1$, there exists $\omega \in K(R)$ such that $a+b \omega \in U(R)$.

(3) Whenever $a, b \in R$ satisfy $a x+b=1$, there exists $y \in R$ such that $a+b y \in U(R)$ and $1-x y \in K(R)$.

Proposition 3.4. Let $R$ be a ring. Then the following statements are equivalent:

(1) Whenever $a, b \in R$ satisfy $a x+b=1$, there exists $\omega \in K(R)$ such that $a+b \omega \in U(R)$.

(2) Whenever $a, b \in R$ satisfy $a x+b=1$, there exists $\omega \in K(R)$ such that $x+\omega b \in U(R)$.

Recall that the opposite ring $R^{o p}$ consists of formal elements $\left\{a^{o p}: a \in R\right\}$ with addition and multiplication given by

$$
a^{o p}+b^{o p}=(a+b)^{o p}, a^{o p} \cdot b^{o p}=(b a)^{o p} .
$$

From Proposition 3.4, we see that $R$ has many full elements if and only if $R^{o p}$ has many full elements. Hence the definition of a ring having many full elements is left-right symmetric.

Proposition 3.5. Let $R$ be a ring. Then $R$ has many full elements if and only if so does $\bar{R}$.

Proof. Assume that $\overline{a x}+\bar{b}=\overline{1}$. Then $a x+b=1+r \in U(R)$ for some $r \in J(R)$, which implies that $a x(1+r)^{-1}+b(1+r)^{-1}=1$. By the virtue of Proposition 3.3, there exists $\omega \in K(R)$ such that $a+b(1+r)^{-1} \omega \in U(R)$. Thus $\bar{a}+\overline{b(1+r)^{-1} \omega} \in U(\bar{R})$ with $\overline{(1+r)^{-1} \omega} \in K(\bar{R})$ by Lemma 3.1. Hence $\bar{R}$ has many full elements by Proposition 3.3. 
Conversely, Given $a x+b=1$ in $R$. There exists $\bar{\omega} \in R / J(R)$ such that $\bar{a}+\overline{b \omega}=\bar{u} \in R / J(R)$. Thus $a+b \omega=u+r \in U(R)$ for some $r \in J(R)$, where $\omega$ is in $K(R)$ by Lemma 3.1, as required.

Example 3.6. Let $R$ be a local ring with $2 \bar{\epsilon} J(R)$. Then $R$ has many full elements.

Proof. $\bar{R}=R / J(R)$ is a division ring since $R$ is local. By the last proposition, it suffices to show that $\bar{R}$ has many full elements. Given that $a x+b=1$ in $\bar{R}$. If $x \neq 0$, we have $a+b x^{-1}=x^{-1} \in U(\bar{R})$, as desired. If $x=0$, then $b=1$. If $a \neq 1$, then $a+b(1-a)=1 \in U(\bar{R})$ with $(1-a) \in U(\bar{R}) \subseteq K(\bar{R}) ;$ If $a=1$, then $a+b \cdot 1 \in U(\bar{R}) \subseteq K(\bar{R})$ by assumption. Hence we complete the proof.

Theorem 3.7. The following are equivalent for any ring $R$ :

(1) $R$ has many full elements.

(2) Whenever $a R+b R=d R$ with $a, b, d \in R$, there exist $u \in U(R)$ and $\omega \in K(R)$ such that $a u+b \omega=d$.

(3) Whenever $a_{1} R+\cdots+a_{m} R=d R$ with $m \geq 2, a_{1}, \ldots, a_{m}, d \in R$, there exist $u \in U(R)$ and $\omega_{2}, \ldots, \omega_{m} \in K(R)$ such that $a_{1} u_{1}+a_{2} \omega_{2}+\cdots+a_{m} \omega_{m}=d$.

Proof. Both $(2) \Rightarrow(1)$ and $(3) \Rightarrow(2)$ are obvious.

$(1) \Rightarrow(2)$ Since $R$ has many full elements, then $R$ has stable range one by definition. Given $a R+b R=d R$ with $a, b, d \in R$, the sets $\{a, b\}$ and $\{d, 0\}$ generate the same $R$-submodule of $R^{2}$. Therefore there exists $U=\left(u_{i j}\right) \in$ $G L_{2}(R)$ such that $(a, b)=(d, 0) U$ by Chen [9, Lemma 2.1].

Obviously, $u_{11} R+u_{12} R=R$. There exists some $\omega \in K(R)$ such that $u_{11}+u_{12} \omega=u \in U(R)$, which implies that $a+b \omega=d v$, hence $a v^{-1}+b \omega v^{-1}=d$ with $v^{-1} \in U(R)$ and $\omega v^{-1} \in K(R)$ by Lemma 3.1, as desired.

$(2) \Rightarrow(3)$ Given $a_{1} R+\cdots+a_{m} R=d R$ with $m \geq 2, a_{1}, \ldots, a_{m}, d \in R$. If $m=$ 2 , the result follows from (2). Assume that the result holds for $m \leq k(k \geq 2)$. Let $m=k+1$, there exist $x_{1}, \ldots, x_{k+1} \in R$ such that $a_{1} x_{1}+\cdots+a_{k+1} x_{k+1}=d$, which implies that $a_{1} R+\cdots+a_{k-1} R+\left(a_{k} x_{k}+a_{k+1} x_{k+1}\right) R=d R$. Then $a_{1} u_{1}+a_{2} \omega_{2}+\cdots+\left(a_{k} x_{k}+a_{k+1} x_{k+1}\right) \omega_{k}=d$ for some $u_{1} \in U(R), \omega_{2}, \ldots, \omega_{k} \in$ $K(R)$. This implies that $\left(a_{1} u_{1}+a_{2} \omega_{2}\right) R+\cdots+a_{k} R+a_{k+1} R=d R$. Thus we have $\left(a_{1} u_{1}+a_{2} \omega_{2}\right) v_{1}+\cdots+a_{k} v_{k-1}+a_{k+1} v_{k}=a_{1} u_{1} v_{1}+a_{2} \omega_{2} v_{1}+\cdots+$ $a_{k} v_{k-1}+a_{k+1} v_{k}=d$ for some $v_{1} \in U(R), v_{2}, \ldots, v_{k} \in K(R)$. Note that $u_{1} v_{1} \in U(R), \omega_{2} v_{1}, v_{2}, \ldots, v_{k} \in K(R)$, thus we complete the proof.

Observe that $R$ has many full elements if and only if so does the opposite ring $R^{o p}$ of $R$, hence the following statement is immediate.

Corollary 3.8. Let $R$ be a ring. Then the following are equivalent:

(1) $R$ has many full elements.

(2) Whenever $R a_{1}+\cdots+R a_{m}=R d$ with $m \geq 2, a_{1}, \ldots, a_{m}, d \in R$, there exist $u_{1} \in U(R)$ and $\omega_{2}, \ldots, \omega_{m} \in K(R)$ such that $u_{1} a_{1}+\omega_{2} a_{2}+\cdots+\omega_{m} a_{m}=d$. 
Next, we investigate the behavior of rings having many full elements under change of rings. Chen [8, Theorem 2.2] showed that if $R$ satisfies a unit 1stable range then so does the $n \times n$ matrix $\operatorname{ring} M_{n}(R)$ for any $n \geq 1$. Let $T$ denote the Morita Context $(A, B, V, W, \psi, \phi)$ as defined in Section 1. Chen [6, Theorem 4] also showed that if $A$ and $B$ have many full elements, so does $T$. It is shown that the analogous results also hold for rings having many full elements.

Theorem 3.9. If a ring $R$ has many full elements, then so does $M_{n}(R)$ for any $n \geq 1$.

Proof. Given $B C+D=I_{n}$ in $M_{n}(R)$, then

$$
A=\left(\begin{array}{cc}
B & D \\
-I_{n} & C
\end{array}\right) \in G L_{2 n}(R) \text { with inverse } A^{-1}=\left(\begin{array}{cc}
C & C B-I_{n} \\
I_{n} & B
\end{array}\right) \text {. }
$$

Let $A=\left(A_{i j}\right)(1 \leq i, j \leq 2)$ with all $A_{i j}=\left(a_{s t}^{i j}\right) \in M_{n}(R)(1 \leq s, t \leq n)$. Then there exists $\xi_{1}=\left(x_{1}, \ldots, x_{n}, y_{1}, \ldots, y_{n}\right)^{T} \in R^{2 n \times 1}$ such that $\mathrm{A} \xi_{1}=$ $(1,0, \ldots, 0)^{T}$, or equivalently,

$$
\begin{gathered}
a_{11}^{11} x_{1}+\cdots+a_{1 n}^{11} x_{n}+a_{11}^{12} y_{1}+\cdots+a_{1 n}^{12} y_{n}=1 \\
\cdots \\
a_{n 1}^{11} x_{1}+\cdots+a_{n n}^{11} x_{n}+a_{n 1}^{12} y_{1}+\cdots+a_{n n}^{12} y_{n}=0 \\
a_{11}^{21} x_{1}+\cdots+a_{1 n}^{21} x_{n}+a_{11}^{22} y_{1}+\cdots+a_{1 n}^{22} y_{n}=0 \\
\cdots \\
a_{n 1}^{21} x_{1}+\cdots+a_{n n}^{21} x_{n}+a_{n 1}^{22} y_{1}+\cdots+a_{n n}^{22} y_{n}=0
\end{gathered}
$$

By Proposition 3.4, we can find some $z_{1} \in R$ such that $a_{11}^{11}+a_{12}^{11} x_{2} z_{1}+\cdots+$ $a_{1 n}^{11} x_{n} z_{1}+a_{11}^{12} y_{1} z_{1}+\cdots+a_{1 n}^{12} y_{n} z_{1}=u_{1} \in U(R)$ and $1-x_{1} z_{1}=\omega_{1} \in K(R)$.

Therefore we obtain that

$$
\begin{gathered}
A\left(\begin{array}{ccccccc}
1 & 0 & \cdots & 0 & 0 & \cdots & 0 \\
x_{2} z_{1} & 1 & \cdots & 0 & 0 & \cdots & 0 \\
\vdots & \vdots & \ddots & \vdots & \vdots & \ddots & \vdots \\
x_{n} z_{1} & 0 & \cdots & 1 & 0 & \cdots & 0 \\
y_{1} z_{1} & 0 & \cdots & 0 & 1 & \cdots & 0 \\
\vdots & \vdots & \ddots & \vdots & \vdots & \ddots & \vdots \\
y_{n} z_{1} & 0 & \cdots & 0 & 0 & \cdots & 1
\end{array}\right) \\
=\left(\begin{array}{ccccccc}
u_{1} & a_{12}^{11} & \cdots & a_{1 n}^{11} & a_{11}^{12} & \cdots & a_{1 n}^{12} \\
a_{21}^{11} \omega_{1} & a_{22}^{11} & \cdots & a_{2 n}^{11} & a_{21}^{12} & \cdots & a_{2 n}^{12} \\
\vdots & \vdots & \ddots & \vdots & \vdots & \ddots & \vdots \\
a_{n 1}^{11} \omega_{1} & a_{n 2}^{11} & \cdots & a_{n n}^{11} & a_{n 1}^{12} & \cdots & a_{n n}^{12} \\
a_{11}^{21} \omega_{1} & a_{12}^{21} & \cdots & a_{1 n}^{21} & a_{11}^{22} & \cdots & a_{1 n}^{22} \\
\vdots & \vdots & \ddots & \vdots & \vdots & \ddots & \vdots \\
a_{n 1}^{21} \omega_{1} & a_{n 2}^{21} & \cdots & a_{n n}^{21} & a_{n 1}^{22} & \cdots & a_{n n}^{22}
\end{array}\right) .
\end{gathered}
$$


Moreover, we get

$$
[*, *] A[*, *] B_{21}(*)=\left(\begin{array}{ccccccc}
u_{1} & a_{12}^{11} & \cdots & a_{1 n}^{11} & a_{11}^{12} & \cdots & a_{1 n}^{12} \\
0 & b_{22}^{11} & \cdots & b_{2 n}^{11} & b_{21}^{12} & \cdots & b_{2 n}^{12} \\
\vdots & \vdots & \ddots & \vdots & \vdots & \ddots & \vdots \\
0 & b_{n 2}^{11} & \cdots & b_{n n}^{11} & b_{n 1}^{12} & \cdots & b_{n n}^{12} \\
a_{11}^{21} \omega_{1} & a_{12}^{21} & \cdots & a_{1 n}^{21} & a_{11}^{22} & \cdots & a_{1 n}^{22} \\
\vdots & \vdots & \ddots & \vdots & \vdots & \ddots & \vdots \\
a_{n 1}^{21} \omega_{1} & a_{n 2}^{21} & \cdots & a_{n n}^{21} & a_{n 1}^{22} & \cdots & a_{n n}^{22}
\end{array}\right) .
$$

Similarly, we can find $\xi_{2}=\left(x_{1}^{\prime}, \ldots, x_{n}^{\prime}, y_{1}^{\prime}, \ldots, y_{n}^{\prime}\right)^{T} \in R^{2 n \times 1}$ such that

$$
[*, *] A[*, *] B_{21}(*) \xi_{2}=(0,1,0, \ldots, 0)^{T},
$$

and we can find some $z_{2} \in R$ such that

$0 \times z_{2}+b_{22}^{11}+b_{23}^{11} x_{3}^{\prime} z_{2}+\cdots+b_{2 n}^{11} x_{n}^{\prime} z_{2}+b_{12}^{12} y_{1}^{\prime} z_{2}+\cdots+b_{2 n}^{12} y_{n}^{\prime} z_{2}=u_{2} \in U(R)$, and $1-x_{2}^{\prime} z_{2}=\omega_{1} \in K(A)$. Thus we have

$$
\begin{aligned}
& {[*, *] A[*, *] B_{21}(*)[*, *] B_{21}(*)=} \\
& \left(\begin{array}{cccccccc}
u_{1} & * & * & \cdots & * & a_{12}^{11} & \cdots & a_{12}^{1 n} \\
0 & u_{2} & * & \cdots & * & b_{21}^{12} & \cdots & b_{2 n}^{12} \\
0 & 0 & c_{33}^{11} & \cdots & c_{3 n}^{11} & c_{31}^{12} & \cdots & c_{3 n}^{12} \\
\vdots & \vdots & \vdots & \ddots & \vdots & \vdots & \ddots & \vdots \\
0 & 0 & c_{n 3}^{11} & \cdots & c_{n n}^{11} & c_{n 1}^{12} & \cdots & c_{n n}^{12} \\
a_{11}^{21} \omega_{1} & a_{12}^{21} \omega_{2} & a_{13}^{21} & \cdots & a_{1 n}^{21} & a_{11}^{22} & \cdots & a_{1 n}^{22} \\
\vdots & \vdots & \vdots & \ddots & \vdots & \vdots & \ddots & \vdots \\
a_{n 1}^{21} \omega_{1} & a_{n 2}^{21} \omega_{2} & a_{n 3}^{21} & \cdots & a_{n n}^{21} & a_{n 1}^{22} & \cdots & a_{n n}^{22}
\end{array}\right) .
\end{aligned}
$$

Likewise, there exist $u_{3}, \ldots, u_{n} \in U(R), \omega_{3}, \ldots, \omega_{n} \in K(R)$ such that

$$
\begin{aligned}
A[*, *] B_{21}(*)= & \left(\begin{array}{cccccccc}
u_{1} & * & * & \cdots & * & a_{12}^{11} & \cdots & a_{12}^{1 n} \\
0 & u_{2} & * & \cdots & * & b_{21}^{12} & \cdots & b_{2 n}^{12} \\
0 & 0 & u_{3} & \cdots & * & c_{31}^{12} & \cdots & c_{3 n}^{12} \\
\vdots & \vdots & \vdots & \ddots & \vdots & \vdots & \ddots & \vdots \\
0 & 0 & 0 & \cdots & u_{n} & d_{n 1}^{12} & \cdots & d_{n n}^{12} \\
a_{11}^{21} \omega_{1} & a_{12}^{21} \omega_{2} & a_{13}^{21} \omega_{3} & \cdots & a_{1 n}^{21} \omega_{n} & a_{11}^{22} & \cdots & a_{1 n}^{22} \\
\vdots & \vdots & \vdots & \ddots & \vdots & \vdots & \ddots & \vdots \\
a_{n 1}^{21} \omega_{1} & a_{n 2}^{21} \omega_{2} & a_{n 3}^{21} \omega_{3} & \cdots & a_{n n}^{21} \omega_{n} & a_{n 1}^{22} & \cdots & a_{n n}^{22}
\end{array}\right) \\
= & \left(\begin{array}{cc}
A_{1} & A_{2} \\
A_{3} & A_{4}
\end{array}\right),
\end{aligned}
$$

where $A_{i}^{\prime} s(1 \leq i \leq 4) \in M_{n}(R)$ are corresponding matrix blocks. Let

$$
E=A_{22}+\operatorname{diag}\left(\omega_{1}, \ldots, \omega_{n}\right) A_{1}^{-1} \times A_{2} .
$$


Since $[*, *] A[*, *] B_{21}(*) \in G L_{2 n}(R)$, we get that $E \in G L_{n}(R)$ and

$$
[*, *] A[*, *] B_{21}(*)=[*, *] B_{21}(W) B_{12}(*),
$$

where $W=-E^{-1} \operatorname{diag}\left(\omega_{1}, \omega_{2}, \ldots, \omega_{n}\right) \in K\left(M_{n}(R)\right)$ by Lemma 3.1. Noting that for any $x \in M_{n}(R),[*, *] B_{21}(x)=B_{21}(y)[*, *]$ for some $y \in M_{n}(R)$, we claim that there exists a $W^{\prime} \in K\left(M_{n}(R)\right)$ such that

$$
B_{21}\left(W^{\prime}\right)\left(\begin{array}{cc}
B & D \\
-I_{n} & C
\end{array}\right)=[*, *] B_{12}(*) B_{21}(*) .
$$

Hence $C+W^{\prime} D \in G L_{n}(R)$. In view of Proposition 3.3, $M_{n}(R)$ has many full elements.

Corollary 3.10. Every $n \times n$ matrix $A$ over $R$ which has many full elements is the sum of an invertible matrix and a full matrix.

Proof. $R$ has many full elements, so does $M_{n}(R)$ by Theorem 3.9. Since $A M_{n}(R)+I_{n} M_{n}(R)=M_{n}(R)$, there exists some $W^{\prime} \in K\left(M_{n}(R)\right)$ such that $A+I_{n} W=U \in G L_{n}(R)$. Hence $A=-W+U$ and the result follows.

Let $T$ denote the Morita Context $(A, B, V, W, \psi, \phi)$ as defined in Section 1.

Theorem 3.11. If the rings $A$ and $B$ have many full elements, so does $T$.

Proof. Assume that $F=\left(\begin{array}{cc}b_{1} & n_{1} \\ m_{1} & b_{2}\end{array}\right), C=\left(\begin{array}{cc}c_{1} & n_{2} \\ m_{2} & c_{2}\end{array}\right)$, and $D=\left(\begin{array}{cc}d_{1} & n \\ m & d_{2}\end{array}\right)$ satisfy $F C+D=\left(\begin{array}{ll}1 & 0 \\ 0 & 1\end{array}\right)$ in $T$. Hence

$G=\left(\begin{array}{ccc}F & D \\ -\left(\begin{array}{ll}1 & 0 \\ 0 & 1\end{array}\right) & C\end{array}\right)=\left(\begin{array}{cc}C & C B-\left(\begin{array}{ll}1 & 0 \\ 0 & 1\end{array}\right) \\ \left(\begin{array}{ll}1 & 0 \\ 0 & 1\end{array}\right) & F\end{array}\right)^{-1} \in G L_{2}(T)$.

Thus there are $x_{1} \in A, x_{2} \in M, y_{1} \in A, y_{2} \in M$ such that

$$
\begin{aligned}
b_{1} x_{1}+\psi\left(n_{1}, x_{2}\right)+d_{1} y_{1}+\psi\left(n_{2}, y_{2}\right) & =1, \\
m_{1} x_{1}+b_{2} x_{2}+m_{2} y_{1}+d_{2} y_{2} & =0, \\
-x_{1}+\psi\left(0, x_{2}\right)+c_{1} y_{1}+\psi\left(n, y_{2}\right) & =0 \\
0 \cdot x_{1}-x_{2}+m y_{1}+c_{2} y_{2} & =0 .
\end{aligned}
$$

Since $A$ has many full elements by assumption, we can find $z_{1} \in A$ such that $b_{1}+\psi\left(n_{1}, x_{2}\right) z_{1}+d_{1} y_{1} z_{1}+\psi\left(n_{2}, y_{2}\right) z_{1}=u_{1} \in U(A)$ and $1-x_{1} z_{1} \in K(A)$ by Proposition 3.4. So we obtain that

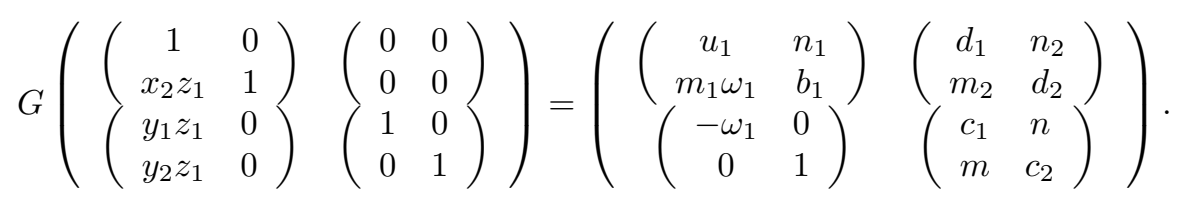


Likewise, we can find $m_{2}^{\prime} \in M, d_{2}^{\prime} \in B, u_{2} \in U(B)$ and $\omega_{2} \in K(B)$ such that

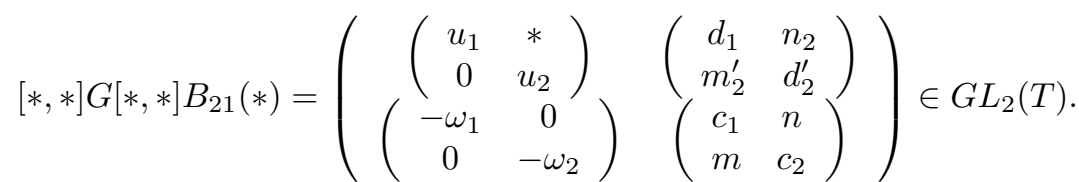

Let

$$
E=\left(\begin{array}{cc}
c_{1} & n \\
m & c_{2}
\end{array}\right)-\left(\begin{array}{cc}
\omega_{1} & 0 \\
0 & \omega_{2}
\end{array}\right)\left(\begin{array}{cc}
u_{1} & * \\
0 & u_{2}
\end{array}\right)^{-1}\left(\begin{array}{cc}
d_{1} & n_{2} \\
m_{2}^{\prime} & d_{2}^{\prime}
\end{array}\right)
$$

Since $[*, *] G[*, *] B_{21}(*) \in G L_{2}(T)$, we have $E \in U(T)$. Then

$$
[*, *] G[*, *] B_{21}(*)=[*, *] B_{21}(W) B_{12}(*)
$$

with $W=-E^{-1} \operatorname{diag}\left(\omega_{1}, \omega_{2}\right) \in K(T)$.

Hence we have $W^{\prime} \in K(T)$ such that

$$
B_{21}\left(W^{\prime}\right)\left(\begin{array}{cc}
F & D \\
-I_{n} & C
\end{array}\right)=[*, *] B_{12}(*) B_{21}(*) .
$$

Therefore, $C+W^{\prime} D \in U(T)$. In view of Proposition 3.3, we conclude that $T$ has many full elements.

Acknowledgment. The authors are grateful to the referee for his/her careful reading of the paper and for the helpful suggestions which improved this paper.

\section{References}

[1] D. D. Anderson and V. P. Camillo, Commutative rings whose elements are a sum of a unit and idempotent, Comm. Algebra 30 (2002), no. 7, 3327-3336.

[2] P. Ara, The exchange property for purely infinite simple rings, Proc. Amer. Math. Soc. 132 (2004), no. 9, 2543-2547.

[3] P. Ara, K. R. Goodearl, and E. Pardo, $K_{0}$ of purely infinite simple regular rings, $K-$ Theory 26 (2002), no. 1, 69-100.

[4] V. P. Camillo and D. Khurana, A characterization of unit regular rings, Comm. Algebra 29 (2001), no. 5, 2293-2295.

[5] V. P. Camillo and H. P. Yu, Exchange rings, units and idempotents, Comm. Algebra 22 (1994), no. 12, 4737-4749.

[6] H. Chen, Morita contexts with many units, Comm. Algebra 30 (2002), no. 3, 1499-1512.

[7] — Generalized stable regular rings, Comm. Algebra 31 (2003), no. 10, 4899-4910.

[8] _ Units, idempotents, and stable range conditions, Comm. Algebra 29 (2001), no. $2,703-717$

[9] - On stable range conditions, Comm. Algebra 28 (2000), no. 8, 3913-3924.

[10] H. Chen and F. Li, Rings with many unit-regular elements, Chinese J. Contemp. Math. 21 (2000), no. 1, 33-38.

[11] K. R. Goodearl and P. Mental, Stable range one for rings with many units, J. Pure Appl. Algebra 54 (1988), no. 2-3, 261-287.

[12] A. Haghany, Hopficity and co-Hopficity for Morita contexts, Comm. Algebra 27 (1999), no. 1, 477-492.

[13] J. Han and W. K. Nicholson, Extensions of clean rings, Comm. Algebra 29 (2001), no. 6, 2589-2595. 
[14] W. K. Nicholson, Lifting idempotents and exchange rings, Trans. Amer. Math. Soc. 229 (1977), 269-278.

[15] _ Strongly clean rings and Fitting's lemma, Comm. Algebra 27 (1999), no. 8, 3583-3592.

[16] W. K. Nicholson and Y. Zhou, Rings in which elements are uniquely the sum of an idempotent and a unit, Glasg. Math. J. 46 (2004), no. 2, 227-236.

[17] G. Xiao and $\mathrm{W}$. Tong, $n$-clean rings and weakly unit stable range rings, Comm. Algebra 33 (2005), no. 5, 1501-1517.

[18] H. Yu, On quasi-duo rings, Glasgow Math. J. 37 (1995), no. 1, 21-31.

[19] Y. Ye, Semiclean rings, Comm. Algebra 31 (2003), no. 11, 5609-5625.

\section{BingJun Li}

Hunan Institute of Humanities Science and Technology

Loudi, Hunan 417000, P. R. China

E-mail address: ydlbj@yahoo.com.cn

LiANGGUi FENG

Department of Mathematics and Systems Science

National University of Defense Technology

Changsha, Hunan 410073, P. R. China 